\title{
Effective properties of the octet-truss lattice material
}

\author{
V.S. Deshpande, N.A. Fleck*, M.F. Ashby \\ Department of Engineering, Cambridge University, Trumpington Street, Cambridge CB2 1PZ, UK \\ Received 11 July 2000; received in revised form 4 January 2001
}

\begin{abstract}
The effective mechanical properties of the octet-truss lattice structured material have been investigated both experimentally and theoretically. Analytical and FE calculations of the elastic properties and plastic yielding collapse surfaces are reported. The intervention of elastic buckling of the struts is also analysed in an approximate manner. Good agreement is found between the predictions of the strength and experimental observations from tests on the octet-truss material made from a casting aluminium alloy. Moreover, the strength and stiffness of the octet-truss material are stretching-dominated and compare favourably with the corresponding properties of metallic foams. Thus, the octet-truss lattice material can be considered as a promising alternative to metallic foams in lightweight structures. (c) 2001 Elsevier Science Ltd. All rights reserved.
\end{abstract}

Keywords: Lattice materials; Collapse surfaces; Porous solids; A. Buckling; C. Finite elements

\section{Introduction}

Over the past few years, a variety of metallic and polymeric foams have been produced for a wide range of potential applications such as the cores of sandwich panels and various automotive parts. A typical aim is to develop lightweight structures that are adequately stiff and strong. Numerous studies on metallic and polymer foams have shown that the strength of the foams is governed by cell wall bending for all loading conditions and scales as $\bar{\rho}^{1.5}$, where $\bar{\rho}$ is the relative density of the foam; see Gibson and Ashby (1997). On the other hand, the strength of a structure that deforms by cell wall stretching scales with $\bar{\rho}$. Thus, for a relative density of $\bar{\rho}=0.1$ the stretching-governed structure is expected to be about three times as strong as the bending governed structure. The aim of this study is to investigate the mechanical

\footnotetext{
* Corresponding author. Tel.: +44-01223-332650; fax: +44-01223-332662.

E-mail address: naf1@eng.cam.ac.uk (N.A. Fleck).
} 
properties of a stretching-governed cellular material and compare them with those of traditional foamed materials.

Deshpande et al. (2001) have recently analysed the criteria for the construction of stretching-dominated cellular materials. A sufficient condition for the deformation of a periodic structure to be stretching dominated is that the unit cell of the structure satisfies Maxwell's criterion for static determinacy. This criterion in three dimensions is given by

$$
b-3 j+6 \geq 0
$$

where $b$ and $j$ are the number of struts and nodes, respectively, in the unit cell. It identifies several classes of unit cells from which stretching-dominated cellular materials (referred to as lattice materials in the following) can be synthesised. In order to give a more definite prescription for constructing lattice materials, Deshpande et al. also analysed a special class of structures with nodes which are all similarly situated nodes are said to be similarly situated if the remainder of the structure appears the same and in the same orientation when viewed from any of the nodes. For this case they showed that the necessary and sufficient condition for the structure to be stretching dominated is that the connectivity at each node is $Z=12$ (or $Z=6$ if the material is two dimensional).

Recent developments in manufacturing techniques have allowed for the manufacture of lattice materials at length scales ranging from millimetres to tens of centimetres. For example, the injection moulding of polymeric structures and subsequent assembly into complex lattice materials is a cheap way to manufacture materials whose constituent struts have aspect ratios less than about 5. These polymeric materials can then be used as sacrificial patterns for investment casting of metallic lattice materials. Rapid prototyping techniques can be used to fabricate materials with lattice parameters on the order of $0.5 \mathrm{~mm}$. Recently, Brittain et al. (2001) have reported an electro-deposition technique to manufacture truss structures with strut diameters as small as $50 \mu \mathrm{m}$.

Along with advances in manufacturing methods for these materials, efforts are underway to investigate their mechanical properties. Wallach and Gibson (2001) have recently reported a combined experimental and finite element (FE) investigation of the strength and stiffness of a truss plate. They find that the properties compare favourably with those of metallic foams. Wicks and Hutchinson (2001) show that optimised truss panels are exceptionally weight-efficient for carrying bending and compression loads, as compared to alternatives such as honeycomb core sandwich panels or stringer stiffened plates. Although the properties of truss plates have been analysed, analytical studies on the properties of full $3 \mathrm{D}$ lattice materials are lacking.

In this paper we shall investigate the properties of the octet-truss (Fuller, 1961) lattice material. The nodes of the octet-truss are configured in a "face centred cubic" arrangement, such that each node has a similar situation and a connectivity of $Z=12$. We report analytical and FE calculations of the elastic-plastic properties as well as collapse surfaces due to elastic buckling. The predictions are compared with experimental observations from tests on an octet-truss material made from an aluminium casting alloy (LM25). 


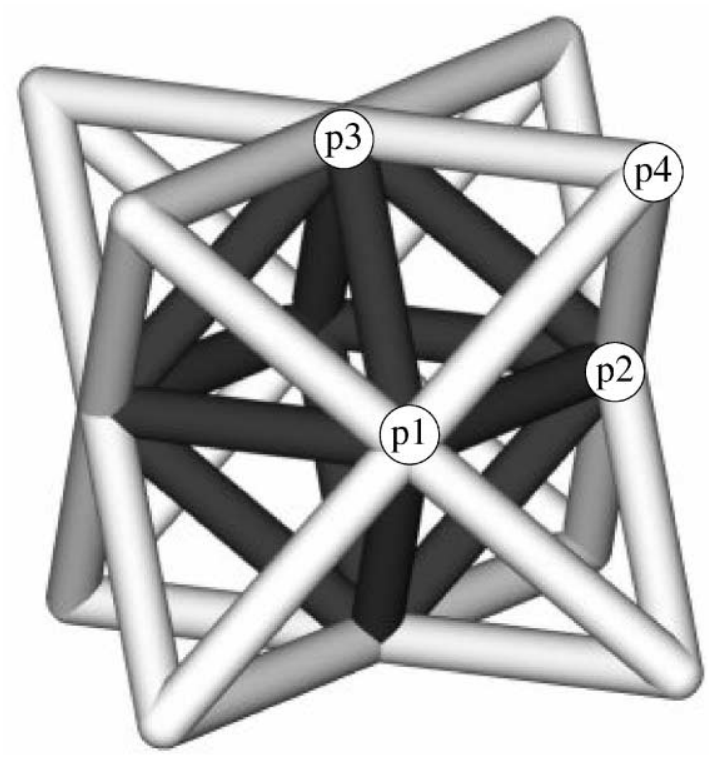

Fig. 1. Structure of the octet-truss lattice material. The darkened struts represent a octahedral cell while the nodes labelled p1-p4 form a tetrahedral cell.

\subsection{Description of microstructure}

A unit cell of the lattice structure is sketched in Fig. 1 and clearly shows its FCC nature. Octahedral cells can be stacked to synthesise the octet-truss structure, with each strut of an octahedral cell shared between two neighbouring cells. Thus, for the purposes of structural calculations it suffices to analyse the octahedral cell. An isometric sketch of a typical octahedral cell, with the associated Cartesian co-ordinate system $(x, y, z)$ is shown in Fig. 2.

An alternative repeating unit which can be stacked in the same orientation to construct the octet-truss lattice material is a regular tetrahedron. One such tetrahedral cell with nodes labelled $\mathrm{p} 1-\mathrm{p} 4$ is shown in Fig. 1 . An isometric sketch of this tetrahedral cell with the associated global co-ordinate system $(1,2,3)$ of the octet-truss is included in Fig. 2. Here, the 1-2 plane is parallel to the base plane p1-p2-p3 and the two-axis is parallel to the strut between nodes $\mathrm{p} 2$ and $\mathrm{p} 3$. The $(1,2,3)$ co-ordinate system corresponds to the three-fold symmetry of the octet-truss material about the three-axis. Note that the 1-2 plane is a close-packed plane of the FCC structure and constitutes a fully triangulated layer in the lattice material. Thus, the octet-truss material can be constructed by the successive packing of the triangulated layers in "ABCABC..." positions with each layer separated by a tetrahedral core.

In the remainder of this paper an octet-truss lattice material constructed from identical circular cylindrical struts is analysed. The conclusions drawn can easily be generalised for the case of hollow or solid struts of arbitrary cross-section. The relative density 


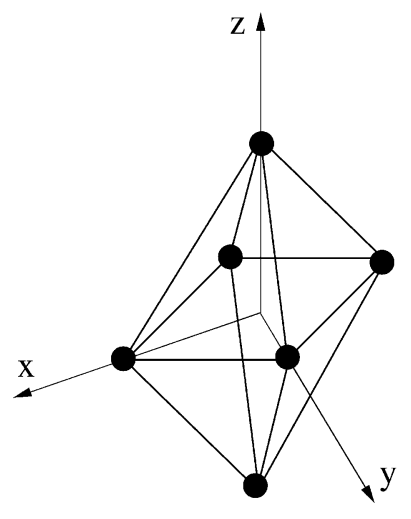

Octahedral cell

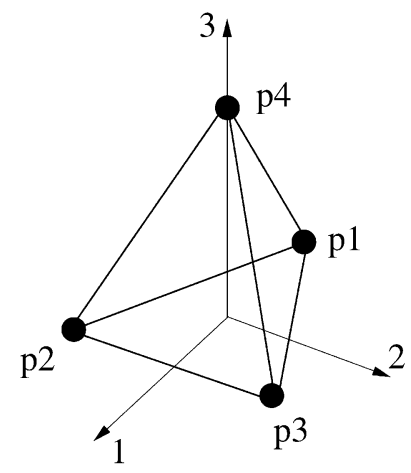

Tetrahedral cell

Fig. 2. Isometric sketches of the octahedral and tetrahedral cells with the associated co-ordinate systems.

$\bar{\rho}$ of the octet-truss lattice material (ratio of the density of the lattice material to the density of the solid material from which it is made) is given by

$$
\bar{\rho}=6 \sqrt{2} \pi\left(\frac{a}{l}\right)^{2}
$$

where $a$ and $l$ are the radius and length of a strut, respectively. This formula is a first-order approximation and overestimates the relative density due to double counting of the volume of the nodes. A higher-order approximation of the relative density is given by

$$
\bar{\rho}=6 \sqrt{2} \pi\left(\frac{a}{l}\right)^{2}-C\left(\frac{a}{l}\right)^{3},
$$

where $C$ depends on the detailed geometry of the nodes. For small $a / l$, the first-order approximation suffices and is used in the remainder of the analysis presented in this paper.

\section{Effective elastic properties}

The cubic symmetry of the octet-truss lattice material dictates that the form of the linear elastic stress versus strain relationship is

$$
\left(\begin{array}{l}
\varepsilon_{x} \\
\varepsilon_{y} \\
\varepsilon_{z} \\
\varepsilon_{y z} \\
\varepsilon_{x z} \\
\varepsilon_{x y}
\end{array}\right)=\left(\begin{array}{cccccc}
s_{1} & -s_{2} & -s_{2} & 0 & 0 & 0 \\
& s_{1} & -s_{2} & 0 & 0 & 0 \\
& & s_{1} & 0 & 0 & 0 \\
& \multicolumn{2}{c}{\text { sym }} & s_{3} & 0 & 0 \\
& & & & s_{3} & 0 \\
& & & & & s_{3}
\end{array}\right)\left(\begin{array}{l}
\sigma_{x} \\
\sigma_{y} \\
\sigma_{z} \\
\sigma_{y z} \\
\sigma_{x z} \\
\sigma_{x y}
\end{array}\right),
$$


where the principal material axes $(x, y, z)$ are defined in Fig. 2 and $s_{1}, s_{2}$ and $s_{3}$ are three independent compliances. Note that an isotropic material has only two independent elastic constants with $s_{3}=2\left(s_{1}+s_{2}\right)$.

For small $a / l$, the contribution to overall stiffness from the bending of the struts is negligible compared to stretching of the struts. Thus, the struts are assumed to be pin-jointed at the nodes, and analytical expressions for the compliances follow as

$$
\begin{aligned}
& \frac{1}{s_{1}}=\frac{2 \sqrt{2} \pi}{3}\left(\frac{a}{l}\right)^{2} E_{\mathrm{s}}=\frac{\bar{\rho}}{9} E_{\mathrm{s}}, \\
& \frac{1}{s_{2}}=2 \sqrt{2} \pi\left(\frac{a}{l}\right)^{2} E_{\mathrm{s}}=\frac{\bar{\rho}}{3} E_{\mathrm{s}}
\end{aligned}
$$

and

$$
\frac{1}{s_{3}}=\frac{\pi}{\sqrt{2}}\left(\frac{a}{l}\right)^{2} E_{\mathrm{s}}=\frac{\bar{\rho}}{12} E_{\mathrm{s}}
$$

where $E_{\mathrm{s}}$ is the Young's modulus of the solid material. The macroscopic elastic stress versus strain relationship in the $(1,2,3)$ co-ordinate system is of practical interest and is obtained by transforming (4):

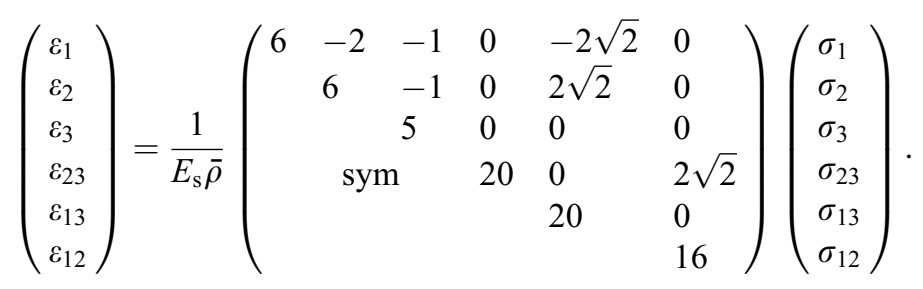

This stress versus strain relationship displays a coupling between the normal components of stress and the shear components of strain in the $(1,2,3)$ co-ordinate system as these directions are not aligned with the principal material directions $(x, y, z)$.

For clarity, in the following we shall refer to the various moduli as either a Young's modulus $E$ or a shear modulus $G$ and subscript them with their respective directions. For example, Young's modulus in the $x$ - and 3-directions will be denoted by $E_{x x}$ and $E_{33}$, respectively, while the shear modulus in the $x-y$ direction will be referred to as $G_{x y}$. It is worth mentioning here that $E_{33}=(\bar{\rho} / 5) E_{\mathrm{s}}$ is the maximum value of Young's modulus of the octet-truss lattice material over all orientations.

\subsection{Comparison with the FE predictions}

The accuracy of the approximate analytical expressions for the moduli was checked against FE calculations performed using the general purpose finite element package ABAQUS (HKS, 1997). In these FE calculations the pin-jointed strut assumption was relaxed. Here, we briefly describe details of the FE calculations.

The octahedral cell with each cylindrical strut modelled by between 20 and 40 Timoshenko beam elements (B32 element of ABAQUS) depending on its length was analysed to extract the three cubic moduli. The displacements of the nodes at the vertices of the cell were constrained so as to prevent rigid-body translation and rotation of 


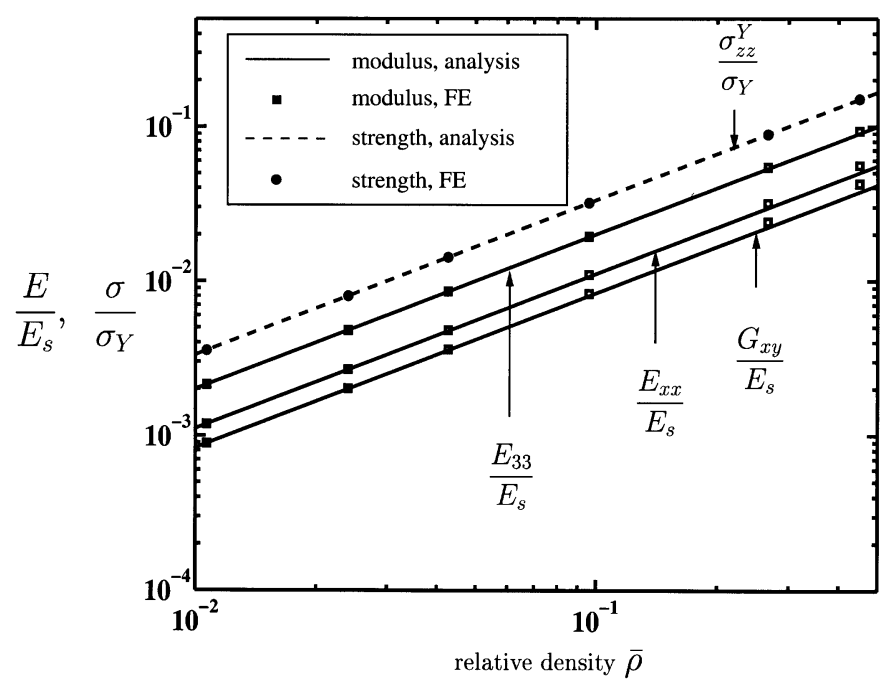

Fig. 3. Comparison of the analytical and FE predictions of the elastic moduli and strength for the octet-truss lattice material.

the cell. Further, the rotations of the nodes at vertices were constrained as dictated by symmetry. Two stress states, uniaxial tension $\sigma_{z z}$ and simple shear $\tau_{x z}$ were prescribed and the moduli extracted from the resulting nodal displacements. We performed calculations for three values of the solid material elastic Poisson's ratio $v_{\mathrm{s}}=0.2,0.3$ and 0.49 . However, $v_{\mathrm{s}}$ had a negligible effect on the octet-truss material moduli. Thus, for the sake of brevity only results for $v_{\mathrm{s}}=0.3$ are presented.

A comparison between the analytical and FE predictions of the moduli $E_{x x}, G_{x y}$ and $E_{33}$ is shown in Fig. 3 for $\bar{\rho}$ ranging from 0.01 to 0.5. Excellent agreement between the $\mathrm{FE}$ and analytical calculations is seen, in support of the pin-jointed strut assumption made in the analytical calculations.

\section{Collapse criteria}

The octet-truss lattice material can fail either by plastic yielding or elastic buckling of the struts. In this section the collapse of the lattice material by these two competing mechanisms is explored. We shall calculate plastic collapse surfaces of the material under various combinations of loading and then proceed to propose an anisotropic yield criterion.

\subsection{Plastic collapse}

In the analytical calculations it is assumed that the struts are pin-jointed and made from a rigid, ideally plastic solid. The macroscopic collapse stress is calculated by equating the external work with the plastic dissipation in stretching the struts for kine- 
matically admissible modes of collapse; that is, an upper bound approach is adopted. The accuracy of these analytical calculations was checked through FE calculations in which the pin-jointed strut assumption was relaxed.

In the FE analysis each cylindrical strut was again modelled by between 20 and 40 Timoshenko beam elements (B32 element of ABAQUS) depending on its length. $J_{2}$ flow theory was employed and the strut material was assumed to be elastic-plastic with the uniaxial stress versus strain law:

$$
\frac{\varepsilon}{\varepsilon_{\mathrm{Y}}}= \begin{cases}\sigma / \sigma_{\mathrm{Y}} & \text { for } \sigma \leq \sigma_{\mathrm{Y}}, \\ \left(\sigma / \sigma_{\mathrm{Y}}\right)^{m} & \text { for } \sigma>\sigma_{\mathrm{Y}},\end{cases}
$$

where $\sigma_{\mathrm{Y}}$ and $\varepsilon_{\mathrm{Y}}$ are the material yield stress and strain, respectively. The elastic Poisson's ratio $v_{\mathrm{s}}$ of the material was assumed to be 0.3 , the yield strain $\varepsilon_{\mathrm{Y}}$ of the strut material was taken equal to $0.1 \%$ and the hardening co-efficient $m=80$ (this small degree of hardening was required to get convergence of the FE calculations). An imperfection in the shape of the plastic buckling mode was imposed on each strut to ensure a unique equilibrium path in the FE calculations. The imperfection is described by the initial transverse deflection $w$ :

$$
w(x)=\frac{\zeta a}{2}\left[1-\cos \left(\frac{2 \pi x}{l}\right)\right],
$$

where $\zeta$ is a dimensionless imperfection parameter, $a$ the strut radius, $l$ the strut length and $x$ the axial co-ordinate along the strut measured from one end. This imperfection is not expected to affect the plastic limit load values; see Section 3.4 for details. For the calculations presented in this section $\zeta$ was taken equal to 0.01 .

The collapse surfaces in $\left(\sigma_{z z}, \sigma_{x z}\right)$ and $\left(\sigma_{x x}, \sigma_{y y}\right)$ space were calculated by analysing the octahedral cell while the tetrahedral cell was used to calculate the collapse surface in $\left(\sigma_{33}, \sigma_{13}\right)$ space. The displacements of the nodes at the vertices of the cell were constrained so as to prevent rigid-body translation and rotation of the cell; and the rotations of the nodes at the vertices were set to zero. The macroscopic yield stresses (defined by the peak of the macroscopic the stress versus strain curve) were calculated for a variety of proportional stress paths and plotted in the relevant stress space to give the plastic collapse surface.

\subsubsection{Collapse surface calculations}

The overall yield surface in macroscopic stress space consists of intersecting collapse surfaces which are associated with particular collapse modes. Plastic strain increments are normal to the relevant collapse surface. In this section we detail calculations of the collapse surfaces for three important practical combinations of macroscopic stressing, viz., $\left(\sigma_{z z}, \sigma_{x z}\right),\left(\sigma_{x x}, \sigma_{y y}\right)$ and $\left(\sigma_{33}, \sigma_{13}\right)$.

3.1.1.1. Collapse surface in $\left(\sigma_{z z}, \sigma_{x z}\right)$ space. The collapse modes for this combination of macroscopic stressing are sketched in side views of the octahedral cell in Fig. 4. In the sketches dashed and solid lines struts are at yield and in the rigid state, respectively, while a solid circle represents a plastic hinge. However, the plastic dissipation at the hinges is neglected in comparison with the dissipation in axial stretching of the yielded 


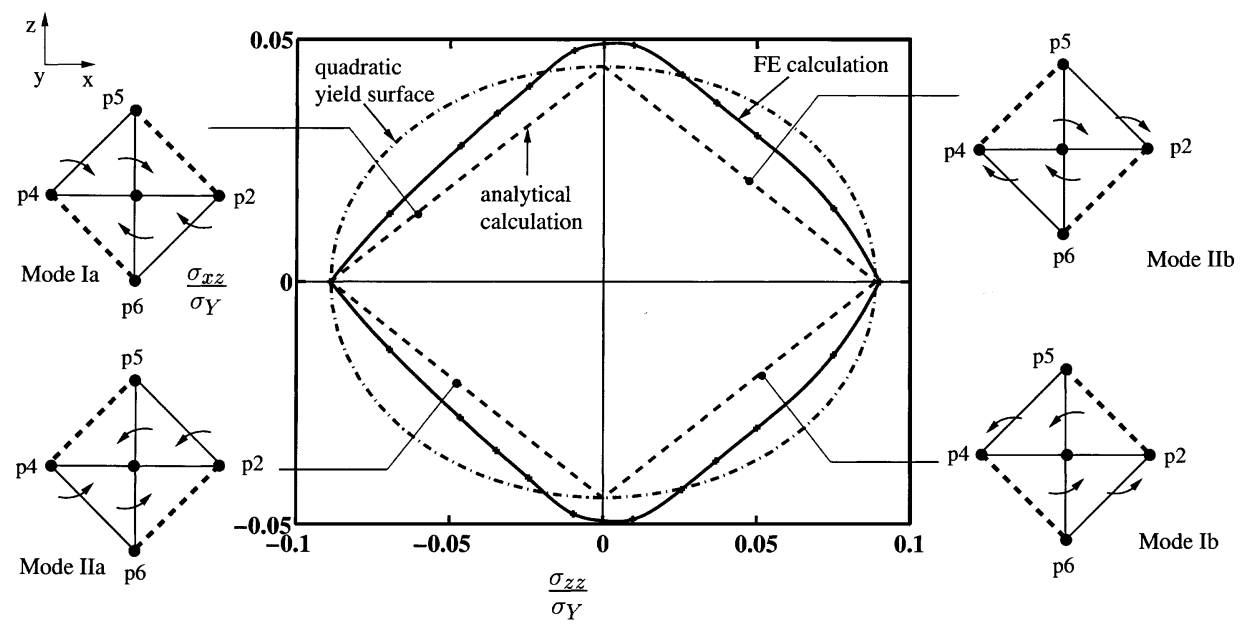

Fig. 4. Comparison between the analytical, FE and proposed yield criterion predictions of the plastic collapse surface in $\left(\sigma_{z z}, \sigma_{x z}\right)$ space $(a / l=0.1)$. In the sketches of the collapse modes, the dashed and solid lines represent struts at yield and in a rigid state, respectively.

struts. As the struts yield either in compression or tension, two collapse locii exist for each yield pattern. Thus, for collapse Mode I, the struts labelled p5-p2, p5-p3, p6-p2 and $\mathrm{p} 6-\mathrm{p} 3$ are yielding and the collapse planes are given by

$$
\text { Mode Ia : } \quad \frac{\sigma_{x z}}{\sigma_{\mathrm{Y}}}=\frac{\sigma_{z z}}{2 \sigma_{\mathrm{Y}}}+\sqrt{2} \pi\left(\frac{a}{l}\right)^{2}
$$

and

$$
\text { Mode } \mathrm{Ib}: \quad \frac{\sigma_{x z}}{\sigma_{\mathrm{Y}}}=\frac{\sigma_{z z}}{2 \sigma_{\mathrm{Y}}}-\sqrt{2} \pi\left(\frac{a}{l}\right)^{2},
$$

where $\sigma_{\mathrm{Y}}$ is the yield stress of the solid material. In Mode II, struts p5-p1, p5-p4, p6 $-\mathrm{p} 1$ and $\mathrm{p} 6-\mathrm{p} 4$ are yielding and the collapse plane equations are

$$
\text { Mode IIa : } \quad \frac{\sigma_{x z}}{\sigma_{\mathrm{Y}}}=-\frac{\sigma_{z z}}{2 \sigma_{\mathrm{Y}}}-\sqrt{2} \pi\left(\frac{a}{l}\right)^{2}
$$

and

$$
\text { Mode IIb : } \quad \frac{\sigma_{x z}}{\sigma_{\mathrm{Y}}}=-\frac{\sigma_{z z}}{2 \sigma_{\mathrm{Y}}}+\sqrt{2} \pi\left(\frac{a}{l}\right)^{2} .
$$

A comparison between the analytical and FE predictions of the uniaxial yield strength $\sigma_{z z}^{\mathrm{Y}}$ is shown in Fig. 3 for $\bar{\rho}$ ranging from 0.01 to 0.5 . The good agreement between the two sets of calculations confirms that the plastic dissipation in the hinges is negligible. FE and analytical calculations of the collapse surface in $\left(\sigma_{z z}, \sigma_{x z}\right)$ space are shown in Fig. 4 for $a / l=0.1$. Again, results from the analytical and FE calculations are in good agreement, and any discrepancy is due to the finite bending strength of the beams in the FE analysis. It is noted from Fig. 4 that the difference between the two predictions varies slightly with loading direction. 


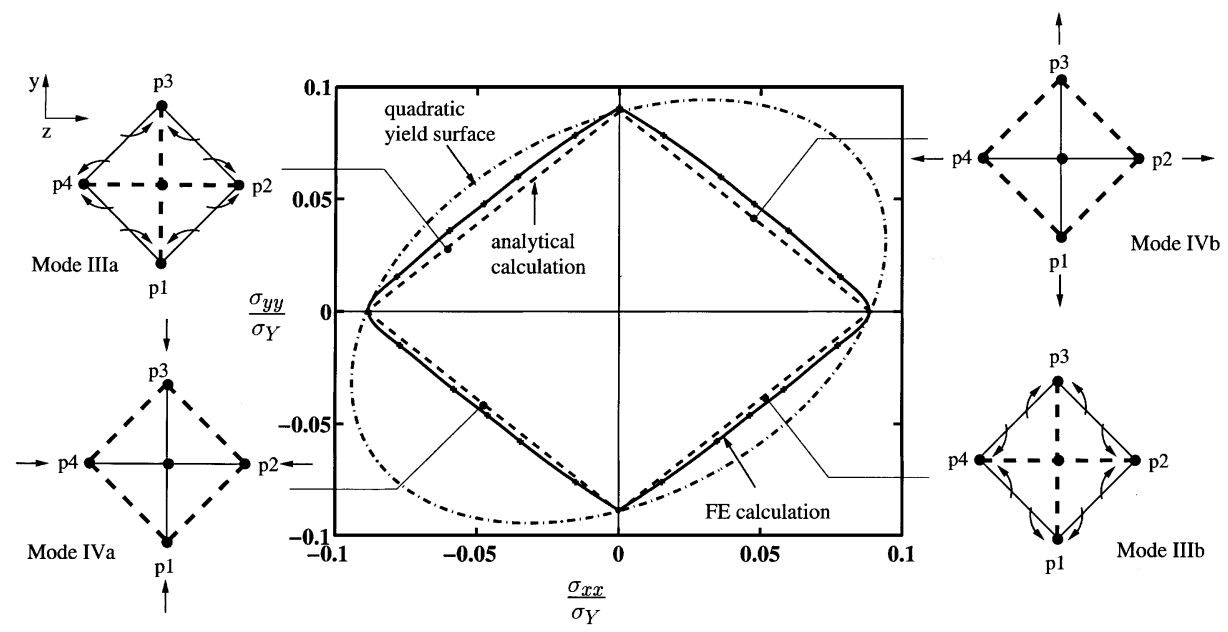

Fig. 5. Comparison between the analytical, FE and proposed yield criterion predictions of the plastic collapse surface in $\left(\sigma_{x x}, \sigma_{y y}\right)$ space $(a / l=0.1)$. In the sketches of the collapse modes, the dashed and solid lines represent struts at yield and in a rigid state, respectively.

The symmetry of the octet-truss lattice material is such that the set of collapse surfaces $\left(\sigma_{m m}, \sigma_{n m}\right)$ are identical, where $m$ and $n$ represent any pair of the $x, y$ or $z$ directions. Further, the octet-truss is periodic with respect to rotations of period $90^{\circ}$ about the $z$-axis. FE calculations show that the shear strength $\sigma_{x z}$ varies by approximately $10 \%$ as the octet-truss is rotated about the $z$-axis with the shear strength being minimum at a $45^{\circ}$ rotation. This suggests that the collapse surface in $\left(\sigma_{z z}, \sigma_{x z}\right)$ space is almost invariant with respect to rotations of the co-ordinate system about the $z$-axis.

3.1.1.2. Collapse surface in $\left(\sigma_{x x}, \sigma_{y y}\right)$ space. The collapse modes under combinations of applied stresses $\left(\sigma_{x x}, \sigma_{y y}\right)$ are sketched in plan views of the octahedral cell in Fig. 5. In Mode III the struts labelled p5-p1, p5-p3, p6-p1 and p6-p3 yield, with the collapse planes described by the equations

$$
\text { Mode IIIa : } \quad \frac{\sigma_{y y}}{\sigma_{\mathrm{Y}}}=\frac{\sigma_{x x}}{\sigma_{\mathrm{Y}}}+2 \sqrt{2} \pi\left(\frac{a}{l}\right)^{2}
$$

and

$$
\text { Mode IIIb : } \quad \frac{\sigma_{y y}}{\sigma_{\mathrm{Y}}}=\frac{\sigma_{x x}}{\sigma_{\mathrm{Y}}}-2 \sqrt{2} \pi\left(\frac{a}{l}\right)^{2} .
$$

In Mode IV, the struts p1-p2, p2-p3, p3-p4 and p1-p4 yield and the collapse planes corresponding to this mode are given by

$$
\text { Mode IVa }: \quad \frac{\sigma_{y y}}{\sigma_{\mathrm{Y}}}=-\frac{\sigma_{x x}}{\sigma_{\mathrm{Y}}}-2 \sqrt{2} \pi\left(\frac{a}{l}\right)^{2}
$$




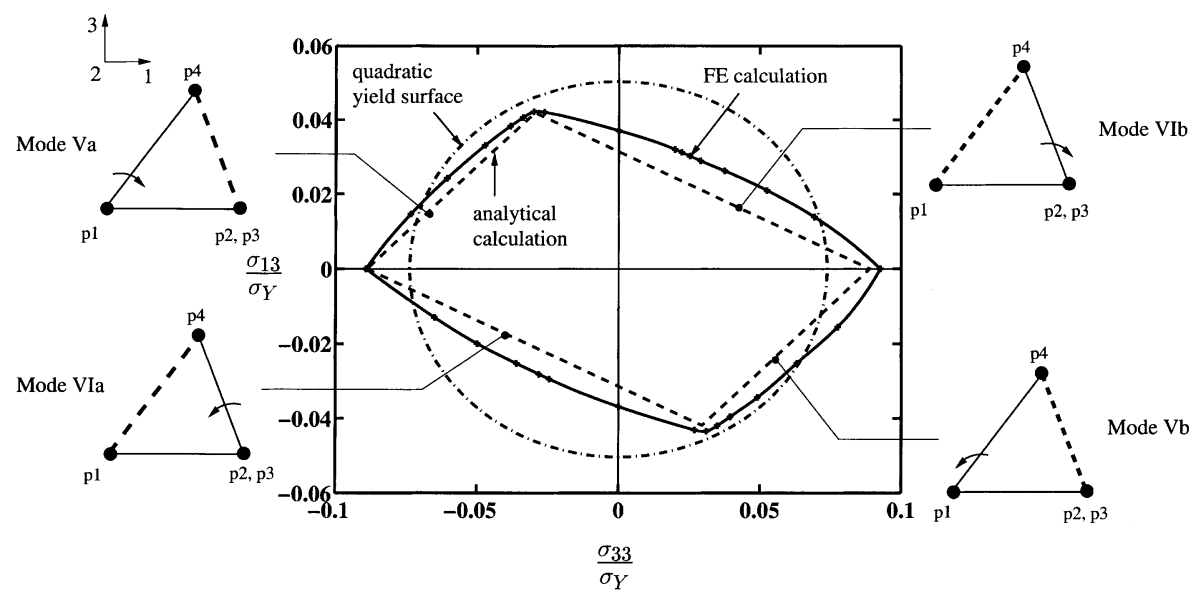

Fig. 6. Comparison between the analytical, FE and proposed yield criterion predictions of the plastic collapse surface in $\left(\sigma_{33}, \sigma_{13}\right)$ space $(a / l=0.1)$. In the sketches of the collapse modes, the dashed and solid lines represent struts at yield and in a rigid state, respectively.

and

$$
\text { Mode IVb : } \quad \frac{\sigma_{y y}}{\sigma_{\mathrm{Y}}}=-\frac{\sigma_{x x}}{\sigma_{\mathrm{Y}}}+2 \sqrt{2} \pi\left(\frac{a}{l}\right)^{2} .
$$

The FE calculations of the collapse surface are included in Fig. 5; good agreement is noted with the above analytical predictions. Note that symmetry dictates that the set of collapse surfaces $\left(\sigma_{m m}, \sigma_{n n}\right)$ are identical where $m$ and $n$ represent any pair of $x, y$ or $z$ directions.

3.1.1.3. Collapse surface in $\left(\sigma_{33}, \sigma_{13}\right)$ space. For the combinations $\left(\sigma_{33}, \sigma_{13}\right)$ of macroscopic stress, it is convenient to analyse the tetrahedral cell. The various collapse modes are sketched in side views of the tetrahedral cell, see Fig. 6. In Mode V, the two struts p4-p2 and p4-p3 yield while in Mode VI the strut p4-p1 yields. The equations of the collapse planes corresponding to these modes are given by

$$
\text { Mode Va : } \quad \frac{\sigma_{13}}{\sigma_{\mathrm{Y}}}=\frac{\sigma_{33}}{\sqrt{2} \sigma_{\mathrm{Y}}}+2 \pi\left(\frac{a}{l}\right)^{2}
$$

and

$$
\text { Mode } \mathrm{Vb}: \quad \frac{\sigma_{13}}{\sigma_{\mathrm{Y}}}=\frac{\sigma_{33}}{\sqrt{2} \sigma_{\mathrm{Y}}}-2 \pi\left(\frac{a}{l}\right)^{2}
$$

for Mode V and

$$
\text { Mode VIa }: \quad \frac{\sigma_{13}}{\sigma_{\mathrm{Y}}}=-\frac{\sigma_{33}}{2 \sqrt{2} \sigma_{\mathrm{Y}}}-\pi\left(\frac{a}{l}\right)^{2}
$$


and

$$
\text { Mode VIb : } \quad \frac{\sigma_{13}}{\sigma_{\mathrm{Y}}}=-\frac{\sigma_{33}}{2 \sqrt{2} \sigma_{\mathrm{Y}}}+\pi\left(\frac{a}{l}\right)^{2}
$$

for Mode VI. Comparisons between the analytical and FE calculations for $a / l=0.1$ are shown in Fig. 6: good agreement again confirms the accuracy of the analytical calculations.

The collapse surface in $\sigma_{33}-\sigma_{13}$ space is approximately invariant with respect to rotations of the co-ordinate system about the three-axis, by the following argument. Consider shear loading with $\sigma_{33}=0$. The shear strength $\sigma_{13}$ of the octet-truss is periodic with respect to rotations of period $60^{\circ}$ about the three-axis; FE calculations reveal that the shear strength $\sigma_{13}$ varies by less than $10 \%$ as the octet-truss is rotated about the three-axis, with the shear strength a maximum for a $30^{\circ}$ rotation.

\subsection{Anisotropic yield criterion}

While the collapse surfaces presented in the previous section are useful for displaying the yield stress under specific load paths, a closed-form expression for the yield surface would be advantageous in summarising the collapse response of the octet-truss lattice material. In this section we shall make use of Hill's (1948) generalisation of the von Mises yield criterion for materials with orthotropic symmetry. An orthotropic material has three mutually perpendicular planes of symmetry at every point, with the intersections of these planes known as the principal axes of anisotropy. With respect to the principal axes of anisotropy $(x, y, z)$, Hill's yield criterion has the form

$$
\Phi \equiv \sigma_{\mathrm{d}}^{2}-1=0,
$$

where the applied macroscopic stress is characterised by the effective stress measure $\sigma_{\mathrm{d}}$ given by

$$
\sigma_{\mathrm{d}}^{2} \equiv A\left(\sigma_{x}-\sigma_{y}\right)^{2}+B\left(\sigma_{y}-\sigma_{z}\right)^{2}+C\left(\sigma_{z}-\sigma_{x}\right)^{2}+D \tau_{x z}^{2}+E \tau_{y z}^{2}+F \tau_{x y}^{2} .
$$

Here, $A-F$ are parameters which characterise the degree of anisotropy.

The octet-truss lattice material also has three mutually perpendicular planes of symmetry passing through every node and the $(x, y, z)$ axes as defined in Fig. 2 correspond to the principal axes of anisotropy. Thus, it is appropriate to consider using Hill's criterion to describe the yielding of this material. However, Hill's anisotropic yield criterion assumes that plastic yielding is not influenced by hydrostatic pressure. This is clearly not the case for the cellular octet-truss lattice material. We therefore modify Hill's criterion by incorporating a mean stress dependence on yielding.

As the simplest extension of Hill's yield criterion we assume that the yield function $\Phi$ of the octet-truss lattice material is quadratic in mean stress $\sigma_{\mathrm{m}}=\sigma_{k k} / 3$, and in the deviatoric stress measure $\sigma_{\mathrm{d}} . \Phi$ is then of the form

$$
\begin{aligned}
\Phi & \equiv \sigma_{\mathrm{d}}^{2}+G \sigma_{\mathrm{m}}^{2}-1 \\
& =A\left(\sigma_{x}-\sigma_{y}\right)^{2}+B\left(\sigma_{y}-\sigma_{z}\right)^{2}+C\left(\sigma_{z}-\sigma_{x}\right)^{2}+D \tau_{x z}^{2}+E \tau_{y z}^{2}+F \tau_{x y}^{2}+G \sigma_{\mathrm{m}}^{2}-1=0,
\end{aligned}
$$


where $A-G$ are material constants. The quadratic term $\sigma_{\mathrm{m}} \sigma_{\mathrm{d}}$ is rejected as it is assumed that the yield stresses under hydrostatic compression and tension are equal. It must be remembered that the yield criterion (16) only has the form shown when the principal axes of anisotropy coincide with the reference axes; otherwise, the form of the yield condition changes in a way that can be found by rotating the co-ordinate system.

It remains to calibrate the yield criterion to determine the material constants $A-G$. The uniaxial and shear yield strengths with respect to the material principal axes and the hydrostatic yield strength $\sigma_{\mathrm{m}}^{\mathrm{Y}}$ of the octet-truss lattice material are

$$
\begin{aligned}
& \sigma_{x x}^{\mathrm{Y}}=\sigma_{y y}^{\mathrm{Y}}=\sigma_{z z}^{\mathrm{Y}}=2 \sqrt{2} \pi\left(\frac{a}{l}\right)^{2} \sigma_{\mathrm{Y}}, \\
& \sigma_{x y}^{\mathrm{Y}}=\sigma_{x z}^{\mathrm{Y}}=\sigma_{y z}^{\mathrm{Y}}=\sqrt{2} \pi\left(\frac{a}{l}\right)^{2} \sigma_{\mathrm{Y}}
\end{aligned}
$$

and

$$
\sigma_{\mathrm{m}}^{\mathrm{Y}}=2 \sqrt{2} \pi\left(\frac{a}{l}\right)^{2} \sigma_{\mathrm{Y}}
$$

respectively. Upon substituting the above yield stresses into (16) and solving for $A-G$, the yield criterion for the octet-truss lattice material can be re-written as

$$
\begin{aligned}
\Phi \equiv & \frac{4}{9}\left[\left(\sigma_{x}-\sigma_{y}\right)^{2}+\left(\sigma_{y}-\sigma_{z}\right)^{2}+\left(\sigma_{z}-\sigma_{x}\right)^{2}\right] \\
& +4\left[\tau_{x z}^{2}+\tau_{y z}^{2}+\tau_{x y}^{2}\right]+\sigma_{\mathrm{m}}^{2}-\left[2 \sqrt{2} \pi\left(\frac{a}{l}\right)^{2} \sigma_{\mathrm{Y}}\right]^{2}=0,
\end{aligned}
$$

where $2 \sqrt{2} \pi(a / l)^{2} \sigma_{\mathrm{Y}}$ is the uniaxial yield strength in a principal direction of anisotropy. Thus, to fully describe the state of anisotropy of the octet-truss lattice material we must know the orientations of the principal axes of anisotropy and the measured uniaxial yield strength in the principal directions.

Comparisons between the calculated collapse surfaces and the predictions of the above yield criterion are shown in Figs. 4-6. In $\left(\sigma_{z z}, \sigma_{x z}\right)$ space, good agreement is seen between the predictions of the proposed yield criterion and both the FE and analytical calculations. On the other hand, in $\left(\sigma_{x x}, \sigma_{y y}\right)$ space the proposed yield criterion substantially overestimates the yield stresses under biaxial tension and compression. In $\left(\sigma_{33}, \sigma_{13}\right)$ space, the yield criterion does not capture the form of the collapse surface accurately. The Hill criterion has been explored in this paper due to its simplicity; however, it is a purely empirical description and there is no a priori reason for it to capture the yield response of the orthotropic octet-truss accurately.

In an effort to propose a better functional form for the yield criterion we tried higher-order functions of $\sigma_{\mathrm{m}}$ and $\sigma_{\mathrm{d}}$ (viz., fourth- and sixth-order functions). However, no improvements in the accuracy of the predictions were found. The authors have been unable to construct a simple expression for a yield criteria that will capture the plastic collapse of the octet-truss lattice material under a variety of stress states to sufficient accuracy. 


\subsection{The elastic buckling strength}

The octet-truss lattice material collapses by elastic buckling of the struts if the Euler buckling load of the struts as given by

$$
P_{\mathrm{E}}=\frac{n^{2} \pi^{3} E_{\mathrm{s}} a^{4}}{4 l^{2}}
$$

is less than their plastic yielding load $P_{Y}=\pi a^{2} \sigma_{Y}$. The factor $n$ in (19) depends upon the rotational stiffness of the end nodes of the strut and is central to the problem of calculating $P_{\mathrm{E}}$ for a given network of struts. When a strut buckles, the rotation of its ends is opposed by the bending of the other struts: they exert a restoring moment and it is this that determines the factor $n^{2}$ in (19). Thus, $n$ depends upon the buckling mode. The cells of the octet-truss lattice material may buckle in many different modes and the resulting problem is very complicated to analyse completely. We simplify the problem by assuming that the struts are pin-jointed. Thus, the rotational stiffness of the nodes is zero and $n=1$ in (19). It is recalled that the buckling load of an axially loaded strut is strongly dependent on the end constraints, and so the calculations presented below should be viewed as lower bounds to the buckling strength.

The collapse stresses due to the elastic buckling of the struts are estimated by equating the external work to the internal work in buckling the struts for kinematically admissible collapse modes. In these analytical calculations we assume the struts are rigid up to the onset of buckling. The post-buckling load-shortening relation for an inextensional pin-ended strut of length $l$ is given by (Budiansky, 1974)

$$
P \approx P_{\mathrm{E}}\left(1+\frac{\Delta}{2 l}\right)
$$

for small axial displacements $\Delta$. Thus, a first-order approximation of the work associated with a shortening $\Delta$ is $P_{\mathrm{E}} \Delta$ and the total internal work is $\sum P_{\mathrm{E}} \Delta$ over all the buckling struts. Note that in the analytical calculations we neglect the effects of elastic deformations prior to the onset of buckling. The accuracy of this assumption has been checked through finite deformation FE calculations.

In the FE calculations, the struts were assumed to be pin-jointed elastic beams comprising of Timoshenko beam elements (B32 of ABAQUS). The strut material was assumed to be elastic-plastic with the uniaxial stress versus strain law (7). Calculations were performed for two material yield strains $\varepsilon_{Y}=0.05$ and 0.1 with a strain hardening exponent $m=80$ in both cases. As in the plastic calculations the octahedral cell was used in $\left(\sigma_{z z}, \sigma_{x z}\right)$ and $\left(\sigma_{x x}, \sigma_{y y}\right)$ collapse surface calculations while the tetrahedral unit cell was employed to calculate the $\left(\sigma_{33}, \sigma_{13}\right)$ collapse surface. An imperfection of shape given by the elastic buckling mode of pin-ended struts, and described by the initial transverse deflection,

$$
w(x)=\zeta a \sin \left(\frac{\pi x}{l}\right)
$$

was imposed on each strut. As in (8), $\zeta$ is the imperfection level and $x$ is the axial co-ordinate along the strut measured from one end. For the calculations presented in this section, an imperfection level $\zeta=0.01$ was added to all the struts. Note that the 


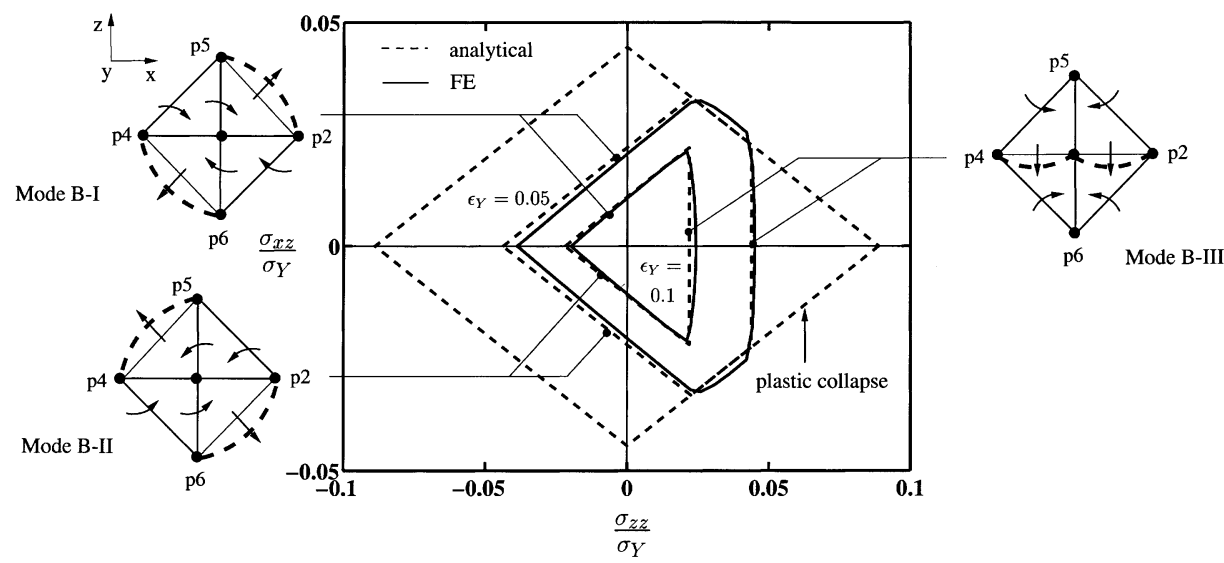

Fig. 7. Comparison between the analytical and FE predictions of the buckling governed collapse surface in $\left(\sigma_{z z}, \sigma_{x z}\right)$ space, for $\varepsilon_{\mathrm{Y}}=0.05$ and $0.1(a / l=0.1)$.

limit load for buckling is insensitive to the choice of $\zeta$ for the material and geometrical parameters considered here; see Section 3.4 for details.

\subsubsection{Buckling collapse surfaces}

We now proceed to detail the collapse surfaces due to elastic buckling, as computed by analytical and FE methods for the combinations of macroscopic stressing considered earlier, $\left(\sigma_{z z}, \sigma_{x z}\right),\left(\sigma_{x x}, \sigma_{y y}\right)$ and $\left(\sigma_{33}, \sigma_{13}\right)$.

3.3.1.1. Collapse surface in $\left(\sigma_{z z}, \sigma_{x z}\right)$ space for elastic buckling. The sketches in Fig. 7 show the possible buckling modes in side views of the octahedral cell, with the dashed lines representing the buckled struts. Since the struts only buckle in compression, a single collapse plane is associated with each buckled state and the equations of the collapse planes for the three collapse modes are given by

$$
\begin{aligned}
& \text { Mode B-I : } \quad \frac{\sigma_{x z}}{\sigma_{\mathrm{Y}}}=\frac{\sigma_{z z}}{2 \sigma_{\mathrm{Y}}}+\frac{\pi^{3}}{2 \sqrt{2} \varepsilon_{\mathrm{Y}}}\left(\frac{a}{l}\right)^{4}, \\
& \text { Mode B-II : } \quad \frac{\sigma_{x z}}{\sigma_{\mathrm{Y}}}=-\frac{\sigma_{z z}}{2 \sigma_{\mathrm{Y}}}-\frac{\pi^{3}}{2 \sqrt{2} \varepsilon_{\mathrm{Y}}}\left(\frac{a}{l}\right)^{4}
\end{aligned}
$$

and

$$
\text { Mode B-III : } \quad \frac{\sigma_{z z}}{\sigma_{\mathrm{Y}}}=\frac{\pi^{3}}{\sqrt{2} \varepsilon_{\mathrm{Y}}}\left(\frac{a}{l}\right)^{4} .
$$

The buckling collapse planes (22) are plotted in Fig. 7 for $a / l=0.1$ and solid material yield strains $\varepsilon_{Y}=0.05$ and 0.1 . The appropriate plastic collapse planes are included in the figure. Good agreement is seen between the FE and analytical calculations in support of the infinitesimal deformation assumption made in the analytical calculations. 


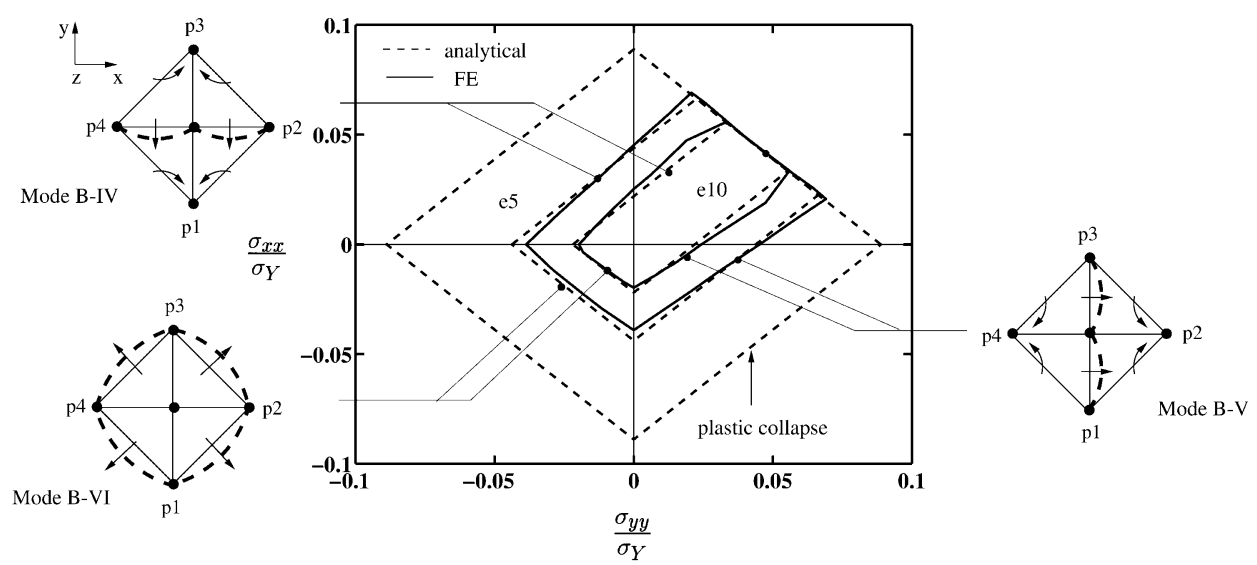

Fig. 8. Comparison between the analytical and FE predictions of the buckling governed collapse surface in $\left(\sigma_{x x}, \sigma_{y y}\right)$ space, for $\varepsilon_{\mathrm{Y}}=0.05$ and $0.1(a / l=0.1)$.

On comparing the buckling collapse stresses (22) with the plastic collapse stresses we note that the entire collapse surface is governed by the elastic buckling of the struts when $\varepsilon_{\mathrm{Y}}>\left(3 \pi^{2} / 4\right)(a / l)^{2}$.

3.3.1.2. Collapse surface in $\left(\sigma_{x x}, \sigma_{y y}\right)$ space for elastic buckling. The buckling collapse modes for the loading in $\left(\sigma_{x x}, \sigma_{y y}\right)$ space are sketched in Fig. 8. The buckled struts are represented by dashed lines in plan views of the octahedral cell. The equations of the collapse planes for the three collapse modes are

$$
\begin{aligned}
& \text { Mode B-IV : } \quad \frac{\sigma_{y y}}{\sigma_{\mathrm{Y}}}=\frac{\sigma_{x x}}{\sigma_{\mathrm{Y}}}+\frac{\pi^{3}}{\sqrt{2} \varepsilon_{\mathrm{Y}}}\left(\frac{a}{l}\right)^{4}, \\
& \text { Mode B-V : } \quad \frac{\sigma_{y y}}{\sigma_{\mathrm{Y}}}=-\frac{\sigma_{x x}}{\sigma_{\mathrm{Y}}}-\frac{\pi^{3}}{\sqrt{2} \varepsilon_{\mathrm{Y}}}\left(\frac{a}{l}\right)^{4}
\end{aligned}
$$

and

$$
\text { Mode B-VI : } \quad \frac{\sigma_{y y}}{\sigma_{\mathrm{Y}}}=\frac{\sigma_{x x}}{\sigma_{\mathrm{Y}}}-\frac{\pi^{3}}{\sqrt{2} \varepsilon_{\mathrm{Y}}}\left(\frac{a}{l}\right)^{4} .
$$

FE and analytical predictions of the collapse surfaces in $\left(\sigma_{x x}, \sigma_{y y}\right)$ space are shown in Fig. 8 for $a / l=0.1$ and $\varepsilon_{Y}=0.05$ and 0.1 ; the predictions of both methods are in good agreement. Elastic buckling collapse governs collapse for all stress states other than a biaxial tensile stress state: the three buckling modes B-IV to B-VI are activated in preference to plastic collapse if $\varepsilon_{Y}>\left(\pi^{2} / 4\right)(a / l)^{2}$. Under biaxial tension, the collapse mode is always by plastic yield irrespective of the value of $\varepsilon_{\mathrm{Y}}$.

3.3.1.3. Collapse surface in $\left(\sigma_{33}, \sigma_{13}\right)$ space for elastic buckling. The collapse modes in $\left(\sigma_{33}, \sigma_{13}\right)$ space are sketched in top and side views of the tetrahedral cell in Fig. 9. 


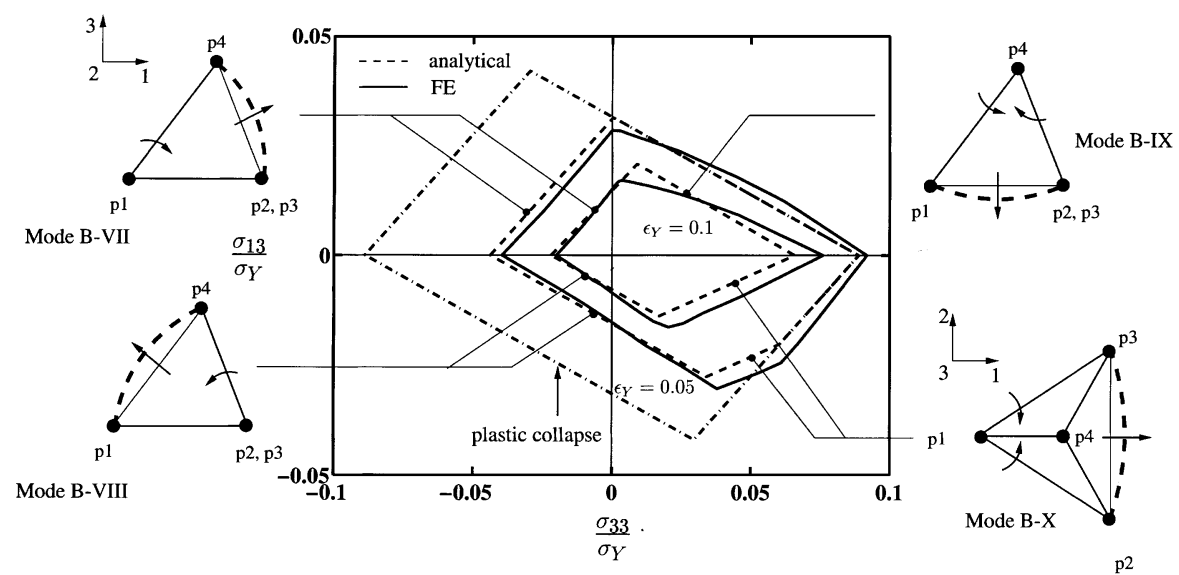

Fig. 9. Comparison between the analytical and FE predictions of the buckling governed collapse surface in $\left(\sigma_{33}, \sigma_{13}\right)$ space, for $\varepsilon_{\mathrm{Y}}=0.05$ and $0.1(a / l=0.1)$.

The equations of the four collapse modes are given by

$$
\begin{aligned}
& \text { Mode B-VII : } \quad \frac{\sigma_{13}}{\sigma_{\mathrm{Y}}}=\frac{\sigma_{33}}{\sqrt{2} \sigma_{\mathrm{Y}}}+\frac{\pi^{3}}{2 \varepsilon_{\mathrm{Y}}}\left(\frac{a}{l}\right)^{4}, \\
& \text { Mode B-VIII : } \quad \frac{\sigma_{13}}{\sigma_{\mathrm{Y}}}=-\frac{\sigma_{33}}{2 \sqrt{2} \sigma_{\mathrm{Y}}}-\frac{\pi^{3}}{4 \varepsilon_{\mathrm{Y}}}\left(\frac{a}{l}\right)^{4}, \\
& \text { Mode B-IX : } \quad \frac{\sigma_{13}}{\sigma_{\mathrm{Y}}}=-\frac{\sigma_{33}}{2 \sqrt{2} \sigma_{\mathrm{Y}}}+\frac{3 \pi^{3}}{4 \varepsilon_{\mathrm{Y}}}\left(\frac{a}{l}\right)^{4}
\end{aligned}
$$

and

$$
\text { Mode B-X : } \quad \frac{\sigma_{13}}{\sigma_{\mathrm{Y}}}=\frac{\sqrt{2}}{5} \frac{\sigma_{33}}{\sigma_{\mathrm{Y}}}-\frac{3 \pi^{3}}{5 \varepsilon_{\mathrm{Y}}}\left(\frac{a}{l}\right)^{4} .
$$

On comparing these elastic buckling collapse stresses with the corresponding plastic collapse stresses (13) and (14), it follows that buckling modes B-VII and B-VIII are activated if $\varepsilon_{\mathrm{Y}}>\left(\pi^{2} / 4\right)(a / l)^{2}$ while modes B-IX and B-X are triggered if $\varepsilon_{\mathrm{Y}}$ exceeds $\left(3 \pi^{2} / 4\right)(a / l)^{2}$ and $\left(7 \pi^{2} / 20\right)(a / l)^{2}$, respectively. Note that the entire collapse surface is governed by the elastic buckling of the struts when $\varepsilon_{\mathrm{Y}}>\left(3 \pi^{2} / 4\right)(a / l)^{2}$.

A comparison between the analytical and FE predictions of the collapse surface in $\left(\sigma_{33}, \sigma_{13}\right)$ space is shown in Fig. 9 for $a / l=0.1$ and $\varepsilon_{Y}=0.05$ and 0.1 . The small discrepancies between the analytical and FE predictions are due to finite deformation effects: as mentioned earlier the analytical calculations for the collapse loads were performed for infinitesimal deformations while the FE analysis was a finite deformation calculation. 


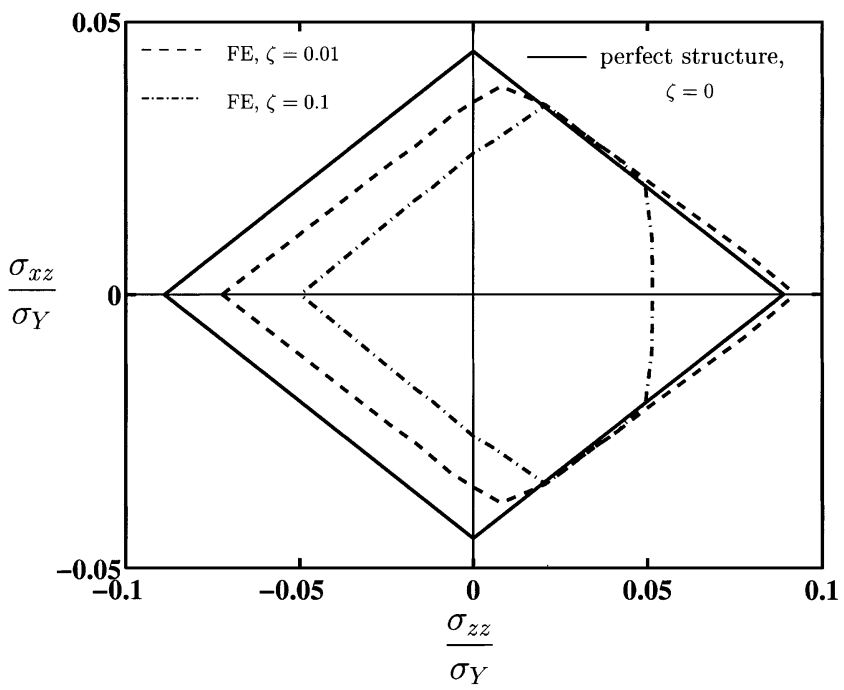

Fig. 10. Effect of imperfections on the collapse surface in $\left(\sigma_{z z}, \sigma_{x z}\right)$ space, for $\varepsilon_{Y}=0.025$ and $a / l=0.1$.

\subsection{Effect of geometric imperfections}

In general, the elastic buckling and plastic collapse surfaces overestimate the collapse stresses of an elasto-plastic lattice material; interactions between the elastic buckling and plastic yielding of the struts substantially knock-down the collapse stresses. In this section we examine this interaction and use the finite element method to investigate the effect of imperfections in the form of strut waviness. For the sake of consistency with the elastic buckling calculations detailed in the previous section we assume that the struts are pin-jointed.

Here we consider imperfections of the same shape as the buckling mode and described by (21). The influence of the imperfection level $\zeta$ on the collapse loads of an axially loaded pin-ended strut is most marked when $\varepsilon_{\mathrm{Y}} \approx(\pi a / 2 l)^{2}$ (Hutchinson, 1974). For example, if $a / l=0.1$ and $\varepsilon_{Y}=0.025$, the collapse loads are expected to be substantially reduced due to the influence of imperfections.

FE calculations of the collapse surface in $\left(\sigma_{z z}, \sigma_{x z}\right)$ space for $a / l=0.1$ and $\varepsilon_{Y}=0.025$ are shown in Fig. 10 for two levels of imperfections, $\zeta=0.01$ and 0.1 . The collapse stresses for both levels of imperfections are substantially lower than those for the perfect structure. In fact, for $\zeta=0.1$ the collapse load of the imperfect strut is about half that of the perfect strut which results in mode B-III becoming active and truncating the tensile side of the plastic collapse surface.

On the other hand, if $\varepsilon_{Y}$ is either much greater than or much less than $(\pi a / 2 l)^{2}$ the collapse load of an axially loaded pin-ended strut is expected to be reasonably insensitive to the imperfection level. FE and analytical calculations of the collapse surfaces in $\left(\sigma_{z z}, \sigma_{x z}\right)$ space for $a / l=0.1$ are shown in Fig. 11 for solid material yield 


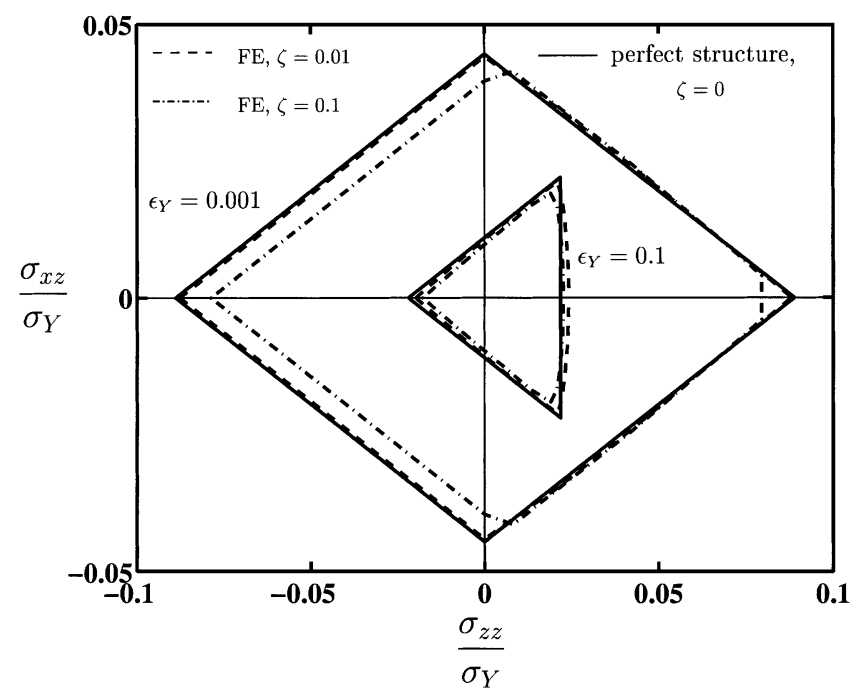

Fig. 11. Effect of imperfections on the collapse surface in $\left(\sigma_{z z}, \sigma_{x z}\right)$ space, for $\varepsilon_{\mathrm{Y}}=0.001$ and $0.1(a / l=0.1)$.

strains $\varepsilon_{Y}=0.001$ and 0.1 . As expected the collapse surface is reasonably insensitive to the imperfection level, with the collapse stresses decreasing by less than $10 \%$ for $\zeta=0.1$. We suggest here that the elasto-plastic collapse stresses of an imperfect octet-truss lattice material idealised as a pin-jointed structure can be estimated by re-calibrating the buckling collapse planes against the axial collapse load of an imperfect pin-ended strut.

\section{Comparison between measured and predicted modulus and yield strength}

We proceed by comparing the predictions detailed in the previous sections with the measured uniaxial compression strength of the octet-truss lattice material made from a casting aluminium alloy (LM25) of composition Al-Si 7-Mg 0.3 (wt\%).

A brief description of the manufacturing route for this lattice material is given below. Triangulated layers with locating holes at the nodes, and tetrahedral cores with locating pins at the nodes, were injection moulded in polystyrene. The octet-truss microstructure was then constructed by adhering the triangulated layers in an "ABCABC..." arrangement with alternating layers of the tetrahedral core. This polystyrene lattice was used as the sacrificial pattern in a "lost-wax" investment casting process to produce the LM25 lattice material. The octet-truss lattice material employed in this study comprised solid cylindrical struts of radius $a=1 \mathrm{~mm}$ and length $l=14 \mathrm{~mm}$, and the overall specimen dimension was $280 \mathrm{~mm} \times 140 \mathrm{~mm} \times 60 \mathrm{~mm}$, with five tetrahedral core layers. A photograph of the lattice material made in LM25 is shown in Fig. 12; the stacking of the five tetrahedral core layers separated by the triangulated layers is clearly seen in this picture. 


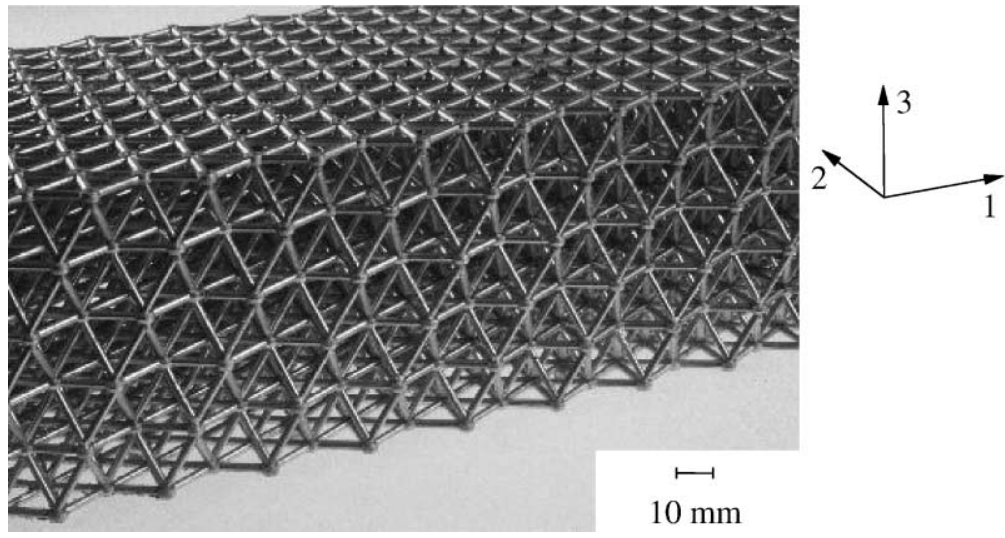

Fig. 12. Photograph of the octet-truss lattice material made from a casting aluminium alloy, LM25.
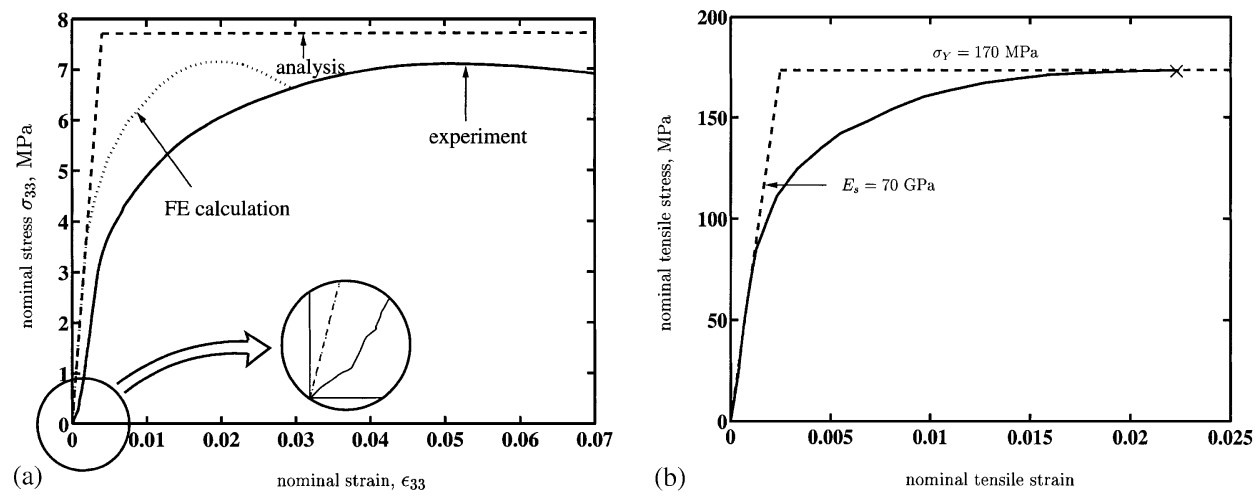

Fig. 13. (a) Comparison between the experimental observations and predictions of the $\sigma_{33}$ versus $\varepsilon_{33}$ response of the LM25 lattice material. (b) Uniaxial tensile response of the as-cast LM25 aluminium alloy.

Uniaxial compression tests in the three-direction were performed using a standard screw driven test machine. The load was measured by the load cell of the test machine and used to define the nominal stress in the specimen. The average nominal strain between two triangulated layers was measured via a clip gauge. The measured uniaxial stress versus strain curve, $\sigma_{33}$ versus $\varepsilon_{33}$, is plotted in Fig. 13a for the nominal strain rate $\dot{\varepsilon}_{33}=10^{-3} \mathrm{~s}^{-1}$. The stress versus strain curve exhibits a hardening response up to a strain of approximately 5\%. Beyond this strain, the response is softening corresponding to plastic buckling of the struts. Bedding-in effects during the early stages of deformation were detected as seen in Fig. 13a. These bedding-in effects occur at the nodes in the lattice material: the pins of the tetrahedral core bed into the holes of the triangulated layers during the initial stages of deformation.

In order to compare the measured and predicted stiffness and strength of the lattice material we measured the uniaxial tensile response of the as-cast LM25 struts; the measured response is plotted in Fig. 13b. It was found that the LM25 can be 


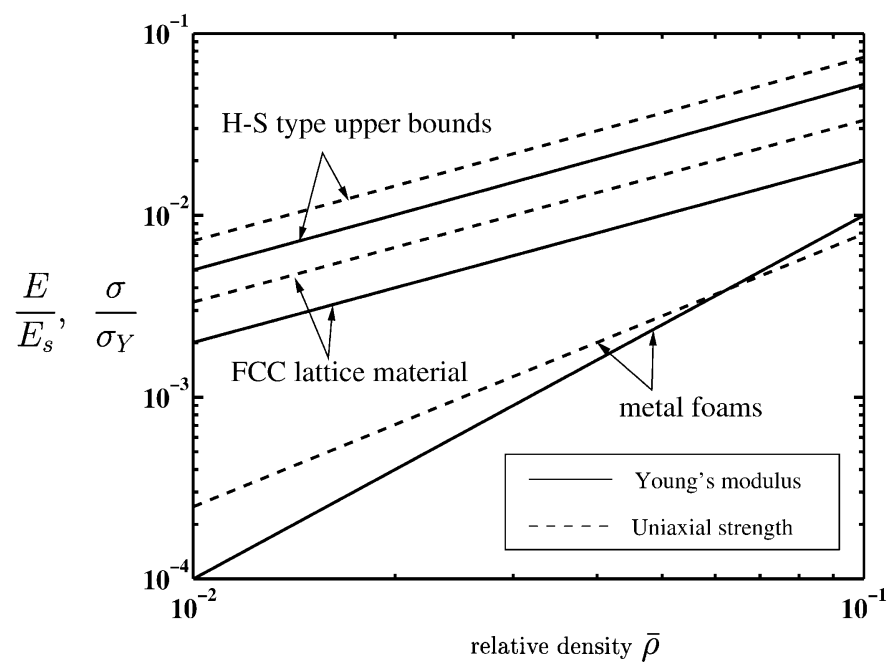

Fig. 14. Comparison between the stiffness and strength of the octet-truss lattice material, metallic foams and the upper bounds for isotropic voided materials.

approximated by an elastic perfectly plastic solid with a Young's modulus $E_{\mathrm{s}}=70$ $\mathrm{GPa}$ and a yield stress $\sigma_{\mathrm{Y}}=170 \mathrm{MPa}$. While these values of $E_{\mathrm{S}}$ and $\sigma_{\mathrm{Y}}$ were used in the analytical predictions, the FE calculations were performed using the measured tensile stress versus strain response of the solid LM25, as given in Fig. 13b. The analytical and FE predictions of the strength of the lattice material are shown in Fig. 13a: they are in good agreement with the experimental data (note that $(\pi a / 2 l)^{2}\left(1 / \varepsilon_{\mathrm{Y}}\right) \approx 5$ and thus the collapse of this material is not expected to be imperfection sensitive). On the other hand, the calculations overestimate the stiffness of the lattice material. This is due to the bedding-in effects at nodes which were neither modelled in the analytical nor the FE calculations.

\section{A comparison between the stiffness and strength of the octet-truss lattice material and other porous materials}

\subsection{Octet-truss lattice material vs. metallic foams}

It is of practical interest to compare the mechanical properties of lattice materials with those of competing materials such as metallic foams. Here, the stiffness and strength of the octet-truss lattice material are compared in Fig. 14 with those of metallic foams, for relative densities $\bar{\rho}$ in the range $0.01-0.1$. The modulus $E_{33}$ and strength $\sigma_{33}^{\mathrm{Y}}$ of the octet-truss lattice material are plotted in Fig. 14, while the experimentally observed isotropic stiffness and strength values for metallic foams

$$
\frac{E}{E_{\mathrm{S}}}=\bar{\rho}^{2}
$$


and

$$
\frac{\sigma}{\sigma_{\mathrm{Y}}}=0.25 \bar{\rho}^{1.5}
$$

are employed; see for example Ashby et al. (2000). Fig. 14 clearly shows that the stiffness and strength of the octet-truss lattice material exceed the corresponding values for metallic foams by a factor between 3 and 10 .

\subsection{Octet-truss lattice material versus optimal microstructures}

The design aim in the development of the octet-truss lattice material is to maximise the strength (or stiffness) to weight ratio of a nearly isotropic cellular material. To get an estimate of the performance of the lattice material with regards to achieving this goal we compare the properties of the octet-truss lattice material with the upper bounds on the stiffness and strength of isotropic voided materials. The $\mathrm{H}-\mathrm{S}$ (Hashin and Shtrikman, 1963) upper bound on Young's modulus (with $v_{\mathrm{s}}=1 / 3$ ) is shown in Fig. 14 . The figure also contains an upper bound on the uniaxial yield strength, obtained by transforming the linear $\mathrm{H}-\mathrm{S}$ bound to the perfectly plastic case, using the prescription of Suquet (1993). The stiffness and strength of the octet-truss lattice material are seen to be about half the theoretical upper bound values for $\bar{\rho}$ between 0.01 and 0.1 .

A number of classes of two-phase composites are known to attain the $\mathrm{H}-\mathrm{S}$ bounds on the bulk and shear moduli. Readers are referred to Sigmund (1999) for details on these optimal microstructures. Here, we briefly review some of the microstructural features of those optimal composites. Norris (1985) and Milton (1986) proposed differential schemes for constructing composite structures with the extremal $\mathrm{H}-\mathrm{S}$ bulk and shear moduli. While Milton (1986) used a laminate microstructure, Norris (1985) employed a coated sphere architecture. However, the procedures suggested by both these authors are incremental and require an infinite number of mixing processes. Moreover, the procedures do not specifically describe the underlying microstructure of the composite. On the other hand, Francfort and Murat (1986) suggested the so-called "rank" laminates which attain both the bulk and shear $\mathrm{H}-\mathrm{S}$ bounds with a finite number of layering directions. Rank laminates are obtained by a sequential process where at each stage the previous laminate is laminated again with a single lamina (always the same) in a new direction. Thus, a rank- $n$ laminate is produced by $n$ such successive laminations. Francfort and Murat (1986) showed that while in the 2D case, isotropic rank-3 laminates have the extremal bulk and shear moduli, in the $3 \mathrm{D}$ case rank- 6 laminates are the optimal microstructures. Thus, there exist a variety of multi-length-scale microstructures with extremal values of the bulk and shear moduli. However, no single-length-scale microstructure that attains both the bulk and shear $\mathrm{H}-\mathrm{S}$ bounds has been proposed to date.

The bulk and shear moduli of the octet-truss lattice material analysed in this paper are about half the H-S upper bound values. However, the octet-truss lattice with solid struts is a single-length-scale microstructure that can be manufactured relatively easily and cheaply. Further, in contrast to the rank laminates, the octet-truss lattice material has additional potential by virtue of its open structure for multi-functional applications. For example, a sandwich plate with solid skins and the octet-truss lattice core can 
serve as a heat transfer element while simultaneously carrying structural loads. Such applications of open-celled cellular materials are discussed by Evans et al. (1998). Thus, the octet-truss lattice material represents a relatively cheap and weight-efficient structural material with potential multi-functional applications.

\section{Concluding remarks}

The effective mechanical properties of the octet-truss lattice material have been investigated through analytical and FE calculations. Analytical expressions have been derived for the three independent moduli associated with the cubic symmetry of the octet-truss lattice material. Good agreement is observed between the analytical predictions and FE calculations, in support of the assumption that the contribution to the overall stiffness from the bending of the struts is small.

Analytical and FE calculations of the plastic collapse surfaces under three combinations of stressing are presented. Again, good agreement is seen between the analytical and FE calculations. An extension of Hill's anisotropic yield criterion fails to capture the form of the collapse surfaces accurately.

The collapse of the octet-truss lattice material by the elastic buckling of the struts has also been addressed through analytical and FE calculations. To simplify the buckling calculations, the struts are assumed to be pin-jointed. Thus, these calculations are expected to be underestimate the collapse stresses. The collapse surface calculations show that elastic buckling of the struts truncate the plastic collapse surfaces under all combinations of macroscopic stressing other than biaxial tension and hydrostatic tension. Further, the FE calculations confirmed that shape imperfections of the struts knock-down the collapse stresses only when the elastic buckling and plastic yielding loads of the struts are approximately equal.

An octet-truss lattice material manufactured in LM25 aluminium alloy was tested in uniaxial compression in the three-direction. Good agreement is seen between the analytical and FE calculations of the strength and the experimental data. However, the experimentally observed stiffness was lower than the predicted value. This is attributed to the bedding-in of the struts into the nodes during the initial stages of deformation.

The stiffness and strength of the octet-truss lattice material compare favourably with the corresponding properties of metallic foams. In fact, the stiffness and strength values of the octet-truss material are about half the theoretical maximum values for isotropic voided materials: its high strength-to-weight ratio, relative ease of manufacture and potential for multi-functional applications makes the octet-truss lattice material an attractive alternative to metallic foams.

\section{Acknowledgements}

The authors are grateful to DARPA/ONR for their financial support through MURI grant number N00014-1-96-1028 on the Ultralight Metal Structures project at Harvard 
University. The Maudslay research fellowship from Pembroke College, Cambridge provided funding for V.S. Deshpande.

\section{References}

Ashby, M.F., Evans, A.G., Fleck, N.A., Gibson, L.J., Hutchinson, J.W., Wadley, H.N.G., 2000. Metal Foams: A Design Guide. Butterworth Heinemann, Stoneham, MA.

Brittain, S.T., Sugimura, Y., Schueller, J.A., Evans, A.G., Whitesides, G.M., 2001. Fabrication of a mesoscale space-filling truss system using soft lithography and microelectrochemistry. J. Microelectromech. Systems, submitted.

Budiansky, B., 1974. Theory of buckling and post-buckling behaviour of elastic structures. In: Yih, C.-S. (Ed.), Advances in Applied Mechanics, Vol. 14. Academic Press, New York, pp. 2-65.

Deshpande, V.S., Ashby, M.F., Fleck, N.A., 2001. Foam topology: bending versus stretching dominated architectures. Acta Mater., to appear.

Evans, A.G., Hutchinson, J.W., Ashby, M.F., 1998. Multifunctionality of cellular metal systems. Prog. Mater. Sci. 43 (3), 171-221.

Francfort, G., Murat, F., 1986. Homogenization and optimal bounds in linear elasticity. Arch. Rat. Mech. Anal. 94, 307-334.

Fuller, R.B., 1961. Octet truss. U.S. Patent Serial No. 2, 986, 241.

Gibson, L.J., Ashby, M.F., 1997. Cellular Solids: Structure and Properties, 2nd Edition. Cambridge University Press, Cambridge.

Hashin, Z., Shtrikman, S., 1963. A variational approach to the theory of the elastic behaviour of multi-phase materials. J. Mech. Phys. Solids 11, 127-140.

Hill, R., 1948. A theory of the yielding and plastic flow of anisotropic metals. Proc. Roy. Soc. London A 193, 281-300.

HKS, 1997. ABAQUS/Standard Users Manual, Version 5.7. Hibbit, Karlsson and Sorensen Inc., Providence, RI.

Hutchinson, J.W., 1974. Plastic buckling. In: Yih, C.-S. (Ed.), Advances in Applied Mechanics, Vol. 14. Academic Press, New York, pp. 67-144.

Milton, G.W., 1986. Modelling the properties of composites by laminates. In: Ericksen, J.L., Kinderlehrer, D., Kohn, R., Lions, J.-L. (Eds.), Homogenization and Effectie Moduli of Materials and Media. Springer, New York, pp. 150-174.

Norris, A.N., 1985. A differential scheme for the effective moduli of composites. Mech. Mater. 4, 1-16.

Sigmund, O., 1999. A new class of extremal composites. J. Mech. Phys. Solids 48 (2), 397-428.

Suquet, P.M., 1993. Overall potentials and extremal surfaces of power law or ideally plastic composites. J. Mech. Phys. Solids 41 (6), 981-1002.

Wallach, J.C., Gibson, L.J., 2001. Mechanical behaviour of a three-dimensional truss material. Int. J. Solids Struct., submitted.

Wicks, N., Hutchinson, J.W., 2001. Optimal truss plates. Int. J. Solids Struct., submitted. 